DOI: $10.47460 /$ uct.v24i104.369

\title{
REFLEXIONES SOBRE EL FEMINISMO Y LA DIVERSIDAD DE GÉNERO: EL PODER DEL DISCURSO EN LA POLÍTICA PÚBLICA
}

\author{
Villón Rodríguez Nadia Wendoline, Cedeño Astudillo Luis Fernando. \\ nadiavillonr27@hotmail.com, cluis@umet.edu.ec \\ ORCID $^{1}$ : https://orcid.org/0000-0001-5815-6815; \\ ORCID ${ }^{2}$ : https://orcid.org/0000-0002-3513-2746 \\ Universidad Metropolitana del Ecuador \\ Guayaquil-Ecuador
}

Recibido (10/08/20), Aceptado (26/08/20)

\begin{abstract}
Resumen: La igualdad de género es un tema de debate internacional, en él se fundamentan los aspectos de equilibrio e igualdad entre las personas de distinto género. En las últimas décadas se han dado importantes logros en la lucha por la igualdad de género, dando origen a diversas políticas públicas que el Estado desarrolla para constituir a una sociedad más justa y equitativa. Entorno al papel de la mujer en la sociedad y la valoración de los colectivos LGBT se han dado importantes avances sociales, que favorecen la toma de decisiones asertivas y empáticas para aportar soluciones a los problemas de discriminación en todos los contextos socioculturales. En este trabajo se exponen las reflexiones del discurso político en la valoración de la igualdad de género.
\end{abstract}

Palabras Clave: Igualdad de género, justicia social, equidad social, discriminación social.

\section{REFLECTIONS ON FEMINISM AND GENDER DIVERSITY: THE POWER OF DISCOURSE IN PUBLIC POLICY}

\begin{abstract}
Gender equality is a subject of international debate, on which aspects of balance and equality between people of different genders are based. In recent decades there have been important achievements in the fight for gender equality, giving rise to various public policies that the State develops to create a more just and equitable society. Around the role of women in society and the appreciation of LGBT groups, important social advances have been made, which favor assertive and empathic decision-making to provide solutions to discrimination problems in all sociocultural contexts. In this work the reflections of the political discourse in the valuation of gender equality are exposed
\end{abstract}

Keywords: Gender equality, social justice, social equity, social discrimination. 


\section{I.INTRODUCCIÓN}

La expresión justicia de género tiene como propósito eliminar las desigualdades entre las mujeres y los hombres que se producen en la familia, la comunidad, el mercado y el estado [1]. Forma parte del proyecto emancipador de las mujeres y grupos LGBT, en este proyecto se promueven dos principios básicos de justicia que corresponden a la Igualdad, donde se hace énfasis que las perspectivas de género deben incluir todas las identidades y orientaciones sexuales, y por otro lado, la igualdad diferenciada, donde se reconoce el papel de la mujer y los grupos LGBT en las diferentes épocas históricas y sectores de funcionamiento social.

De estos escritos se resalta la responsabilidad del Estado como factor fundamental en la construcción de sociedades igualitarias a través de la implementación de políticas públicas que modulen o direccionen el comportamiento de los ciudadanos para el logro de los objetivos sociales. Esta es una acción clave que debe desempeñar la relación estado/sociedad para lograr que la política pública sea considerada como un motor de cambio y la construcción de la lianza entre organizaciones públicas y privadas, para implementar medidas que influyan en el cambio de conductas relacionadas con la discriminación. De allí que las políticas públicas son consideradas esenciales para promover las revoluciones sociales proyectadas hacia sociedades más justas, pero al mismo tiempo reconocen el inmenso problema sobre discriminación y desigualdad que afectan a las mujeres y a la diversidad de género.

Si bien la tardía tendencia legalista del matrimonio entre personas del mismo sexo en América Latina unió bajo una misma esfera de protección a los grupos LGBT, aún existen importantes desatenciones que marcan una brecha social entre la comunidad transgénero y las demás identidades y orientaciones sexuales. Aunque el llamado matrimonio igualitario representa un progreso en el reconocimiento de sus derechos, la cooperación del poder ejecutivo con los órganos descentralizados, la atención a las necesidades socioeconómicas, demandas relacionadas con el bienestar de la diversidad sexual y la erradicación de la discriminación y segregación laboral, no pueden quedar desapercibidas frente a la globalmente publicitada figura del matrimonio civil [2].

Las últimas décadas han sido claves para el reconocimiento formal de los derechos sexuales en América Latina [3]. Se han generado importantes cambios en relación al derecho de la familia, logrando de esta forma relaciones más igualitarias entre hombres y mujeres dentro del matrimonio, entre las que se incluyen la eliminación de los términos legales entre niños dentro del matrimonio y fuera del matrimonio, así como el re- conocimiento del divorcio en Chile en el año 2004 [4], representando entonces el último país de la región en aceptar las nuevas políticas del matrimonio.

En el año 2009, el matrimonio entre personas del mismo sexo se legalizó en la Ciudad de México, al año siguiente en Argentina y en el año 2019 finalmente la Corte Constitucional aprobó el matrimonio igualitario en el territorio ecuatoriano [5]. La legislación antidiscriminatoria también se ha convertido en un pilar fundamental para la política estatal. En 1998, Ecuador se convirtió en el segundo país del mundo (después de Sudáfrica) en instaurar mecanismos de protección constitucional contra la discriminación por orientación sexual [6], y con la Constitución del año 2008 se convirtió en el primer país de la región en contemplar también la identidad de género por vías supra legales [6]. En el año 2012, siguiendo el Protocolo de Yogyakarta [7], una ley sobre identidad de género en Argentina garantiza a todas las personas el derecho de modificar su carnet de identidad (incluido el sexo registrado en el certificado de nacimiento) para reconocer la identidad de género sin necesidad de intervenciones médicas o de orden legal, garantizando de igual forma el acceso a terapia hormonal y tratamientos quirúrgicos para la reasignación de género.

Pero más allá de los acontecimientos políticos, la Convención Interamericana para Prevenir, Sancionar y Erradicar la Violencia contra la Mujer [8] de forma unánime aprobó en el año 2008 la condena a las violaciones de derechos humanos motivadas por la orientación sexual y la identidad de género [9]. Sin embargo, los alcances de tales reconocimientos han sido visiblemente desiguales, tanto en la protección formal de los derechos sexuales, como en términos del impacto social, aun cuando hay mecanismos de protección legalmente reconocidos. No hace falta entonces indicar que tal ejercicio implica asemejar una diversidad de experiencias con todas las regiones, actores, perspectivas y enfoques metodológicos.

Hay tres ideas centrales podrían enmarcar la discusión sobre el alcance protector de la norma para las personas transgénero; La primera se refiere al momento histórico en el que los movimientos por los derechos sexuales lograron consolidarse, si bien las transiciones han abierto espacios para el diálogo, la adopción de políticas neoliberales que sufrió América Latina [10] en los años 80 como herencia de la intromisión ideológica de Ronald Reagan [11], reforzaron diversas formas de exclusión social para las minorías sexuales. La segunda, implica la dificultad de traducir la identidad de género al limitado lenguaje de la norma y la política pública. Este proceso crea una rigidez de las categorías sociales, 
lo que a su vez puede contribuir a la competencia entre movimientos representantes de minorías y a diversas formas de exclusión que a menudo no se reconocen. $\mathrm{La}$ tercera, se refiere a las brechas políticas que han existido entre los movimientos feministas y grupos LGBT, cuyo estudio nos permite identificar las diferencias y analizar sus puntos en común.

En este trabajo se expone una reflexión sobre la igualdad de género y además se fundamentan los logros alcanzados a lo largo de los últimos años, se enfatiza en el hecho de que las políticas públicas son las que pueden facilitar la valoración de la equidad de género y establecer un discurso a favor de los avances sociales, que harán posible una sociedad más justa.

\section{II.DESARROLLO}

Los países latinoamericanos que surgieron de las guerras de independencia del siglo XIX [12], heredaron estratos sociales divididos en raza, género y clase social. Las prerrogativas económicas, políticas y sexuales de los hombres fueron legalmente sancionadas y protegidas. Las élites políticas consideraban a la familia patriarcal como la base de una sociedad estable y una nación ordenada bajo el mandado de la norma terrenal y divina [13]. Las leyes adaptadas a las tradiciones jurídicas ibéricas y el derecho canónico patrocinaron este ideal y mantuvieron la autoridad del cónyuge sobre la propiedad de sus esposas y la autoridad sobre sus hijas. Se formularon diferencias entre mujeres casadas y solteras, personas con trastornos, mujeres impuras, entre otras categorías. Desde luego, estas restricciones limitaron la autoridad legal de las mujeres casadas sobre sus hijos y la capacidad legal para celebrar contratos, proponer demandas y controlar la propiedad. Las viudas recuperaban el control de su propiedad siempre que no se volvieran a casar, y tanto ellas como las mujeres solteras mayores, podían celebrar contratos y ejercer ciertos otros derechos civiles, aunque no políticos. Los hombres y las mujeres también fueron juzgados de manera diferente según el Derecho Penal [14] (particularmente con los crímenes de honor) que reforzaron los privilegios masculinos y las normas sociales restrictivas que rodean la virtud de las mujeres.

Debido a la débil capacidad del estado y la dependencia de las élites de las sociedades patriarcales, las primeras reformas en el derecho de familia ocurrieron mucho después de la independencia [15]. Los proyectos de modernización comenzaron un proceso fragmentado de laicismo que asumió diversas formas en muchos países latinoamericanos. Las críticas recientes han afrontado los informes teleológicos que postulan las reformas liberales como un simple paso más en el camino progresivo hacia la emancipación de las mujeres, en lugar de señalar sus efectos contradictorios en las relaciones de género, su impacto variable en diferentes grupos minoritarios y sus articulaciones concurrentes con tecnologías de control social en el área de la medicina, salud pública, policía y justicia.

Del mismo modo, si bien la sodomía se despenalizó en la mayor parte de la región [16], [17], las leyes que regulaban la moral, las buenas costumbres, la corrupción de menores, las agresiones a la decencia pública y la ociosidad, permitieron la represión policial continua para mantener el género y el sexo dominante [18]. En términos generales, las élites liberales priorizaron proyectos de modernización sobre las libertades individuales y los ideales igualitarios, estableciendo repúblicas oligárquicas altamente excluyentes. Este es el contexto en el que uno debe comprender los esfuerzos del poder para blanquear a las poblaciones nacionales mediante la atracción de inmigrantes europeos, la eliminación forzada de comunidades indígenas a través de medidas que van desde campañas de genocidio en el Cono Sur hasta la privatización de tierras comunales, así como esfuerzos para regular la prostitución, controlar las enfermedades venéreas y, sobre todo, inocuizar a los desviados sexuales. Inocuo es una persona inofensiva, entonces ¿qué quisieron decir con inocuizar si esta palabra no existe en la lengua española?

Las oligarquías dieron paso a los gobiernos populistas, que desempeñaron un relevante papel transformador en toda la región hasta el siglo XX [19]. Sus líderes eran sujetos que proponían una política inclusiva, algunos con tendencias revolucionarias, otros, con ambiciones más conservadoras [20]. En el plano político, se promulgaron las primeras piezas importantes de legislación laboral y política de bienestar social a medida que reorientaron las economías fuera de los territorios de exportación de materias primas hacia modelos de sustitución de importaciones, industrialización y mercados internos. En los primeros experimentos de movilización masiva, los gobiernos populistas buscaron incorporar las clases medias urbanas y los sectores marginales, estableciendo vínculos corporativos de representación que incluían a los grupos históricamente desprotegidos.

Las mujeres habían participado durante mucho tiempo en actividades sociales relacionadas con la caridad, al tratarse de un papel propio de la extensión natural de la feminidad. Con la expansión de la regulación estatal en esferas nuevas y más íntimas de las relaciones sociales, las mujeres (particularmente de las clases altas) se involucraron en la política de bienestar social [21]. En este contexto, las activistas feministas de la primera 
ola en América Latina, muchas de ellas vinculadas a los procesos socialistas [22], también lograron sus primeros éxitos políticos. Movilizándose de manera paralela a los modos de organizaciones prevalecientes, las mujeres en México organizaron Ligas Feministas con más de cincuenta mil integrantes en el año de 1920, estableciendo estrechos vínculos con el nuevo liderazgo post revolucionario [23]. En Argentina, el Partido Femenino Peronista, fundado en 1949, alcanzó más de medio millón de mujeres inscritas en tres años [24]. Como reflejo de la dimensión transnacional del feminismo, las mujeres reunidas en la Primera Conferencia Feminista de la Liga Panamericana de Mujeres celebrada en la Ciudad de México en 1923, discutieron el acceso al trabajo, los derechos de voto y el control de la natalidad y exigieron un estándar moral único en materia de sexualidad para los hombres y mujeres [25]. A fines de la década de los años 50, el sufragio femenino se reconoció en América Latina a medida que un número creciente de mujeres ingresaba a las universidades y al mercado laboral.

En la década de los años 60 y 70, se produjo una reacción violenta contra el populismo en gran parte de la región, ya que las dictaduras militares y los gobiernos autoritarios trataron de restablecer el orden social jerárquico desafiado por varios actores sociales [26]. Estos regímenes marcaron el comienzo de la represión que diezmó a gran parte de la sociedad civil identificada con el progresismo y la izquierda. El retorno a la democracia en el último cuarto del siglo XX, preparó el escenario para que los movimientos sociales contemporáneos se movilicen en torno a los derechos sexuales que surgieron en el contexto de las aspiraciones democráticas y revolucionarias. De hecho, muchas de las primeras activistas feministas, lesbianas y homosexuales en particular, tenían una historia de militancia en partidos socialistas, grupos estudiantiles radicales y organizaciones revolucionarias. Ellas, al encontrarse frente a una sociedad política dominada por hombres y heterosexista, recurrieron a los movimientos sociales como respuesta al rechazo de los grupos dominantes de la izquierda masculina, que ignoraban sus preocupaciones sobre las relaciones de poder estructuradas en torno al género y la sexualidad. Estas raíces ayudan a explicar la importancia de la política sexual en la región, y los debates sobre la interseccionalidad del feminismo y la liberación sexual con la lucha de clases [26].

En estos acontecimientos es posible observar que la igualdad de género no representaba un tema de interés en la política mundial, y quedaba relegado a un tema de segundo plano. Es entonces en la década de los años 90 que nace el movimiento feminista y LGBT y hacen posible un debate a integrador por parte de la Organiza- ción de las Naciones Unidas.

La mujer representó un papel fundamental en los procesos políticos de igualdad de género. Los grupos feministas pidieron una legislación penal más fuerte contra la violencia sexual y doméstica [10], [26], y la discriminación por orientación sexual, imploraron cambios en el derecho civil para permitir el divorcio y establecer relaciones de género más igualitarias dentro del matrimonio, así como una maternidad libre y voluntaria [27], [4].

Las primeras feministas latinoamericanas estuvieron exclusivamente dedicadas a la lucha contra el patriarcado. Aunque a menudo encontraron resistencia por parte de las feministas heterosexuales, las feministas lesbianas promovieron las discusiones iniciales sobre la importancia de oponerse al estigma sexual. La organización de un grupo de lesbianas en la Primera Conferencia de las Naciones Unidas sobre la Mujer [28] proveyó el único lugar en el foro para estas discusiones donde se debatieron sobre la sexualidad y discriminación por orientación e identidad de género [28]. Con el tiempo, estas nuevas variantes del movimiento feminista establecieron lazos con los nuevos estados democráticos y han luchado por una mayor atención a los problemas de las mujeres y las minorías sexuales, mediante el establecimiento de institutos y programas gubernamentales de mujeres, aun cuando no cuentan con fondos suficientes.

A lo largo del tiempo, un paradigma internacional de salud y diversidad sexual ha logrado una importancia cada vez mayor, a menudo traducido en demandas específicas de políticas públicas para el acceso a servicios de salud, educación y otros de orden social. A la par que se organizaban movimientos feministas, se dieron los movimientos de liberación de homosexuales en Argentina, en el año 1969 [29]. Como lo expresaron los participantes de la primera Marcha del Orgullo Gay y Lésbico celebrada en la Ciudad de México en el año 1979 [30], no debe considerarse la existencia de una liberación política si primero no figura una liberación sexual. Buscando un mayor alcance que otros movimientos sociales al cuestionar la estructuración del binarismo sexual, priorizaron la necesidad de poner fin a la violencia transfóbica, el tratamiento y la prevención del VIH / SIDA, las leyes sobre identidad que permitirían a las personas cambiar su nombre y género en los documentos oficiales, así como un mayor acceso a empleos, educación y atención médica.

También se han establecido organizaciones en América Latina para defender los derechos de las trabajadoras sexuales trans a través de las identidades de género, a manera de activismo reflejado en las distintas estrategias políticas para erradicar las etiquetas sexua- 
les y estigmatización por cuestiones de género. El primer movimiento de este tipo en la región andina, fue la Asociación de Trabajadoras Autónomas del Ecuador, establecida por sexo servidoras en el de 1982 [31]. Esta agrupación estuvo en la palestra pública luego de organizar una huelga donde reclamaron el abuso y la explotación sexual por parte de prostíbulos y los dueños de establecimientos de diversión nocturna. Si bien existen algunos precedentes, el movimiento ha tenido cada vez más simpatizantes de todos los grupos de diversidad sexual, en parte reflejado por sus campañas de prevención del VIH / SIDA y la precariedad laboral. Asimismo, en 1997 [32], y siguiendo el ejemplo de Ecuador, activistas de Costa Rica organizaron una ONG de carácter regional que representa a las trabajadoras sexuales (RedTrabSex) que actualmente tiene afiliaciones en 15 países. Al abrazar un discurso basado en el respeto a los derechos humanos, las activistas han priorizado la no estigmatización del trabajo sexual, el reconocimiento de los derechos laborales para la profesión, la lucha contra el abuso policial y la prevención del VIH / SIDA. Esto ha repercutido en el territorio ecuatoriano, pues, si bien la prostitución no figura como una práctica laboral legalizada por mandato, en el año 2008 se implementó una política que permite a la trabajadora social la inclusión voluntaria al seguro social, lo que le permite acceder a créditos bancarios, viviendas, y demás beneficios que pudiesen existir en el seguro social.

Sin negar las características específicas de cada uno de estos movimientos y las múltiples diferencias entre países, es posible identificar varias tendencias que han distinguido el avance de la política en materia sexual en la región latinoamericana a lo largo de los últimos años. Las ONGs pueden desempeñar un rol importante en la promoción, investigación y prestación de servicios para las comunidades trans. Ellas, mientras juegan un papel cada vez más importante en el activismo latinoamericano para compensar las brechas y limitaciones de la acción estatal en el contexto contemporáneo, buscan democratizar su organización. Ciertamente, los movimientos sociales han mantenido durante mucho tiempo conexiones transnacionales que han tomado diversas formas, aunque el alcance y la frecuencia de tales contactos han aumentado en las últimas décadas. Una expresión particularmente importante de este fenómeno ha sido la organización de conferencias feministas de América Latina y el Caribe [33], celebradas regularmente desde 1981. Estas conferencias han proporcionado un espacio importante para la creación de espacios deliberativos, donde los actores LGBT pueden acumular recursos simbólicos y desarrollar interpretaciones alternativas de identidad y justicia social. Por supuesto, se han producido transformaciones más extensas en las comunidades trans porque se sienten excluidas de la política LGBT generalizada. Esto se refleja, por ejemplo, que los organizadores de las tres primeras conferencias propusieron que las comunidades trans buscaran autofinanciamiento para poder asistir, mientras que, para lo posterior, buscaron fuentes externas de financiación [34].

\section{III.RESULTADOS}

Una vez evaluado diversas fuentes bibliográficas se ha realizado una reflexión sobre la trascendencia de los movimientos feministas en Latinoamérica y su relevancia en el Ecuador. Por ello es posible destacar algunos resultados:

Examinar las intersecciones de la sexualidad y las políticas públicas no es tarea fácil. Un primer examen nos refleja la adopción del discurso como transformador de verdades. El lenguaje se ha convertido en un instrumento de poder, expresado por individuos, grupos y movimientos que trascienden las fronteras. Hoy podemos hablar abiertamente sobre la identidad de género y las personas trans. Sin embargo, esta transformación no se ha desarrollado naturalmente como podría suponer una comprensión ideológica porque existe una confrontación política y social. Más bien, los lenguajes de los derechos se refieren a un proceso histórico incompleto, a través del cual los actores sociales se han unido para redefinir las relaciones sociales, sexuales y de género como una extensión de la ciudadanía. La histórica desigualdad que instituyeron y naturalizaron las jerarquías de género entre hombres y mujeres, se convirtieron en objetos de lucha por la igualdad de derechos de todas las comunidades conectadas con la diversidad sexual. En el curso de estas luchas, nuevos temas sociales han entrado en la escena, desafiando los mismos binarios y categorías de identidad que estructuraron estos conflictos durante mucho tiempo.

El discurso es independiente de los individuos o las instituciones, pero está conformado por un sistema de regulación específica derivados de formaciones discursivas. Para Michel Foucault [35], las reglas de política pública no estarían definidas por nociones tradicionales de soberanía o estado de derecho, sino por un discurso generalizado de quien regula el poder. Él supone que el discurso utilizado en la política pública explora el campo de las prácticas sociales y las disciplinas, exhibiendo se encuentran integrados a través de la costumbres y rituales y valores. Por esta razón, el discurso juega un rol dominador en el ser humano, pues les permiten creer que el sistema se adapta a la sujeción de sus derechos, pero al mismo tiempo consiguen su obediencia. 
La política pública es la principal influencia en los procesos de legitimidad del gobierno para tomar decisiones en nombre de la población y realizar ejercicios de control social. Pero, aunque que el proceso es complejo y las decisiones pueden ser contradictorias, la mera inclusión nominativas de las minorías les otorgan una falsa sensación de bienestar [36]. Por eso, el impacto de las políticas públicas va mucho más allá de las instituciones, pues se dirige a grupos de personas ya dominados. Esto indica que las ideas de Foucault sobre la producción del discurso plantean preguntas sobre las prácticas de gobierno y cómo se forman en oposición a las instituciones históricas. El análisis de los discursos tiene el potencial suficiente para mostrar el vínculo entre la literatura política y la subordinación de las almas. Entonces, el concepto de poder foucaultiano reconoce las diversas influencias de las relaciones sociales más allá de la propia estructura.

Las medidas para abordar la violencia y la discriminación que enfrentan las mujeres y los colectivos LGBT están muy por detrás de los discursos que promueven los organismos gubernamentales. A pesar de las graves violaciones de los derechos humanos, los esfuerzos para proteger a la mujer y a la comunidad LGBT se encuentran todavía en una etapa incipiente.

El desarrollo del feminismo lésbico dejó entrever la conexión entre feminismo y el colectivo LGBT al trabajar directamente contra el sexismo y la homofobia. $\mathrm{Su}$ relevancia en los años ochenta la catapultó como una ideología dominante en cuestiones de género, ofreciendo a las mujeres una nueva forma de pensamiento sobre la sexualidad y el cuerpo, relegando a un segundo plano el argumento simple de la preocupación heterosexual masculina como factor dominante [37].

Referente al delito de violación, las propuestas legislativas se volcaron a enfocar la penetración forzada durante el coito heterosexual donde el hombre perpetra acciones violentas, dado que los círculos feministas mostraron la violencia interpersonal como resultado de manifestación de poder a causa de los hombres, ignorando por completo la violencia sexual dentro de las relaciones homosexuales. Hasta la década de los 80, aunque se visibilizaron grandes logros para las mujeres, el movimiento feminista marginó cuestiones importantes para la comunidad LGBT [38].

\section{IV.CONCLUSIONES}

Una vez terminada la reflexión sobre la igualdad de género y el feminismo en Latinoamérica, es posible concluir que:

A pesar de que existen muchos ejemplos de progreso en materia de Derechos Humanos para las mujeres y colectivos LGBT, gran parte de estos cambios son aislados, no sistémico e insuficientes. Las personas trans continúan viviendo en contextos extremadamente hostiles, al no existir un cambió a gran escala en la materialización de la política pública.

Foucault muestra que los individuos, las instituciones y otros fenómenos sociales están regulados por estos procesos sociales. Los cuerpos y las disciplinas exhiben su regulación por medio de normas de comportamiento, asignación de roles, estructuras y jerarquías que forman parte del proceso de construcción de las políticas públicas. El discurso configura y remodela continuamente la interacción social, y esa interacción no se limita al mundo abarca muchas disciplinas, opiniones ciudadanas, medios de comunicación y actividad política en diferentes escalas espaciales [39].

Las políticas públicas son fundamentales para la generación de una sociedad igualitaria, y deben ir encaminadas a un proceso de equidad sin distinciones de géneros, que permita la estabilidad emocional, psicológica y física de todos los ciudadanos.

Las decisiones de Estado darán pie al respeto de género, a la igualdad de derechos y a la promoción de políticas que beneficien la equidad, que promuevan luna sociedad justa e igualitaria, que se centre en los valores sociales como entes fundamentales de las personas, y que no favorezca la discriminación de las personas.

\section{REFERENCIAS}

[1]UNIFEM, «UNIFEM,» 2010. [En línea]. Available: http://www.ungei.org/MDGBrief-Esp.pdf.

[2]P. Marshall, «Matrimonio entre personas del mismo sexo: una aproximación desde la política del reconocimiento,» Polis (Santiago), pp. 201-230, 2018.

[3]ONU, «Igualdad de Género,» 2019. [En línea]. Available:http://www.inesge.mx/pdf/articulos/igualdad onu.pdf.

[4]C. Loreto, «Divorcio en Chile. Un análisis preliminar tras la nueva Ley de matrimonio civil,» Bibliografía lationoamericana, $\mathrm{n}^{\circ}$ 123, pp. 95-187, 2011.

[5]M. Orellana, «El matrimonio civil igualitario como forma de ejercer el derecho a la igualdad y no discriminación,» Revista de Derecho, pp. 103-121, 2019.

[6]S. Zambrano, «El acceso a la justicia y la tutela judicial efectiva en relación con la seguridad ciudadana en Ecuador,» Tla-melaua, pp. 58-78, 2016.

[7]C. Canevaro, «Los Principios de Yogyakarta en el Sistema Interamericano de Derechos Humanos,» Pontificia Universidad Católica del Perú, Perú, 2020.

[8]L. Mejía, «La Comisión Interamericana de Mujeres,» IIDH, vol. 56, pp. 189-213, 2012. 
[9]O. Alvarez, «El enfoque de género y la violencia contra las mujeres: aproximación al análisis de los conceptos,» Revista Venezolana de Estudios de la Mujer, pp. 45-54, 2006.

[10]L. Frayle, «La experiencia neoliberal de América Latina. Políticas sociales y laborales desde el decenio de 1980,» Revista Internacional del Trabajo, 2009.

[11]M. Sen y G. Velasco, «La administración Reagan y los proyectos de una derecha articulada como respuesta a la crisis norteamericana,» Revista de Ciencias, 1981. [12]M. Chust y J. Serrano, «Presentación. Guerras, monarquía e independencia de la América española,» Asociación de historia contemporánea, $\mathrm{n}^{\mathrm{0}} 74$, pp. 1321, 2009.

[13]J. Bracho, «Narrativa e identidad. El mestizaje y su representación historiográfica,» Identidad y cultura, pp. 55-86, 2009.

[14]T. Pich, «Justicia penal y libertad femenina,» Academia.edu, 2009.

[15]P. Vélez, La historiografía americanista en España, 1755-1936, Madrid: Iberoamericana Vervuert, 2007.

[16]P. Rossi, «Rechazo a la despenalización de la sodomia desde el punto de vista del magisterio de la iglesia y del derecho natural,» Revista chilena de Derecho, vol. 25, no 4, pp. 915-937, 1998.

[17]M. Chamocho, «Sodomía: El crimen y pecado contra natura o historia de una intolerancia,» CCSD, p. 282, 2012.

[18]Amnistía Internacional, Amor, odio y ley: despenalizar la homosexualidad, 2016.

[19]O. González, «Los orígenes del populismo latinoamericano; una mirada diferente,» Cuadernos del cendes, vol. 24, no 66, 2007.

[20]O. González, «Los orígenes del populismo latinoamericano: Una mirada diferente*,» Cuadernos del Cendes, pp. 75-104, 2007.

[21]A. M. Stuven, «La mujer ayer y hoy: un recorrido de incorporación social y política,» CENTRO DE POLÍTICAS PÚBLICAS UC, pp. 1-22, 2019.

[22]A. Restrepo, «Femenismo y discurso de género,» POLIS, no 9, 2019.

[23]y. o. Gabriela Cano, La Revolución de las Mujeres en México, México: Instituto Nacional de Estudios Históricos de las Revoluciones de México (inehrm), 2014. [24]C. Barry, «Eva Perón y la organización política de las mujeres,» Revista Arenal , pp. 1-38, 2001.

[25]G. L. A. Ramírez, «El Primer Congreso Feminista de Yucatán 1916. El camino a la legislación del sufragio y reconocimiento de ciudadanía a las mujeres. Construcción y tropiezos,» Estudios Políticos, pp. 59-89, 2016.

[26]M. Valdivieso, Movimientos de mujeres y lucha fe- minista en América Latina y el Caribe, Buenos Aires: CLACSO, 2016.

[27]P. Almeida, Movimientos sociales en America Latina, Buenos Aires: CLACSO, 2017.

[28]C. Hinojosa, «Gritos y susurros. Una historia sobre la presencia pública de las feministas lesbianas,» Testimonios, pp. 177-186, 2001.

[29]C. Figari, «El movimiento LGBT en América Latina: institucionalizaciones oblicuas,» de Movilizaciones, protestas e identidades políticas en la Argentina del bicentenario, Buenos Aires, Nueva Trilce, 2010, pp. 225-240.

[30]G. d. M. Secretaría de Cultura, «Breve historia de la primera marcha LGBT+ de México,» 26 Junio 2019.

[En línea]. Available: https://www.gob.mx/cultura/es/ articulos/breve-historia-de-la-primera-marcha-lgbttti-de-mexico?idiom=es.

[31]J. Robles, «Derechos de la mujer, moral sexual y prostitución,» de Tercer certamen de ensayo sobre Derechos Humanos, Toluca, Comisión de Derechos Humanos del Estado de México, 2000, pp. 11-41.

[32]M. J. Chávez, «La salud y los derechos humanos de las mujeres trabajadoras sexuales,» Aportes Andinos, pp. 47-59, 2014.

[33]ONU, «XIV Conferencia Regional sobre la mujer de América Latina y el Caribe,» de XIV Conferencia Regional sobre la mujer de América Latina y el Caribe, Santiago, 2020.

[34]C. Bunch, «La travesía de las mujeres esbianas por el feminismo internacional,» mayo 2000. [En línea]. Available: https://cwgl.rutgers.edu/docman/coalition-building-publications/291-lesbianstravel-roadfeminisms-sp-pdf/file.

[35]C. Duarte, «Políticas públicas, un movimiento discursivo: "régimen de verdad" por demostrar,» Revista Tendencias \& Retos, pp. 81-92, 2012.

[36]C. Rúa Delgado, «La legitimidad en el ejercicio del poder político en el estado social de derecho. Una revisión desde el caso colombiano,» Ius et Praxis, pp. 85-122, 2013.

[37]S. Muñoz, Feminismos y LGTB: encuentros y desencuentros., Castellón de la Plana: Universitat Jaume I , 2017.

[38]C. Saldivia, «Violencia íntima en parejas jóvenes del mismo sexo en Chile,» Última Década, pp. 184-212 , 2017.

[39]R. Giraldo, «Poder y resistencia en Michel Foucault,» Tabula Rasa, pp. 103-122, 2006. 